\title{
ESTUDIO DE LA VARIABILIDAD DE LA DENSIDAD BASICA DE LA MADERA DE Pinus taeda PARA PLANTACIONES DE MISIONES Y NORTE DE CORRIENTES
}

Obdulio Pereyra ${ }^{1}$ Mónica Gelid ${ }^{2}$

\begin{abstract}
RESUMEN
El presente trabajo tiene por objetivo evaluar plantaciones de Pinus taeda (Procedencias: Livingston, Marion, Local) a través de la densidad básica en distintas regiones de la Provincia de Misiones y Corrientes, y determinar correlaciones entre la densidad básica obtenida con tarugos y con probetas extraídas de secciones transversales de las toras a distintas alturas del árbol. Fueron estudiadas las diferencias de densidades entre procedencias, edades y regiones, para lo cual se utilizaron 25 árboles de cada punto de muestreo, totalizando 475 árboles.De acuerdo al análisis de los resultados, se pudo observar que la densidad de la madera aumenta con la edad y que depende casi exclusivamente de la proporción de leño temprano y leño tardío. Que en el rango de edades entre 6 - 10 años la densidad básica es mayor para el Livingston que para el Marion, ocurre lo mismo para edades mayores entre 11 - 15 años. Del análisis de la correlación entre tarugos y discos se obtuvieron tres modelos estadísticos que se ajustan adecuadamente.

Palabras clave: Pinus, Densidad básica, Tecnologia
\end{abstract}

\section{RESEARCH OF THE VARIABILITY IN BASIC DENSITY OF Pinus taed $a$ PLANTATIONS FROM MISIONES AND NORTH OF CORRIENTES PROVINCES}

\begin{abstract}
The present research has the aim to evaluate Pinus taeda plantations (origins: Livingstone, Marion, Local) through their basic densities in different regions from Misiones and Corrientes provinces, and to determine correlations between basic density obtained with increment cores and with samples taken from cross section at different heights of the tree. Differences in densities between origins, ages and regions where studied taking 25 trees at each sampling location, totalizing 475 trees.According to analysis of results it was observed that density of wood diminishes with age and depends almost exclusively from the proportion of early wood to late wood. Also in the range of ages between $6-10$ years, basic density is higher for Livingstone than for Marion and the same for higher ages between 11 - 15 years. From correlation analysis between increment cores and round tree sections where obtained three statistic models that adjusted adequately.
\end{abstract}

Key-words: Pinus, Basic density, Tecnology

\section{INTRODUCCIÓN}

Las provincias de Misiones y Corrientes cuentan en la actualidad con extensiones de bosques implantados, en su mayoría con especies exóticas entre las que se mencionan, dentro de las coníferas el género Pinus (Pinus elliottii, Pinus taeda) y el género Araucaria (Araucaria angustifolia); y entre las latifoliadas el género Eucalyptus y otras de importancia económica como la Toona o Cedro australiano (Toona ciliata), el Kiri (Pouwlonia sp) y el Paraiso (Melia azederach).
Estas plantaciones abastecen de
materia prima a las industrias de transformación mecánica (Aserrado y Madera Compensada) e industrias de transformación química (Fábrica de Pastas Celulósicas y Papel) desde comienzo de la década del 60, superando ampliamente a algunas especies nativas, como es el caso del Pino paraná (Araucaria angustifolia), que también se utiliza en reforestaciones.

Los datos que proporciona este trabajo podrían ser una herramienta más, que ayudará a tomar decisiones en cuanto a turnos de corta,

\footnotetext{
${ }^{1}$ Ing. Ftal. M.Sc. Profesor. Director del Departamento De Tecnología De La Madera E-mail opereyra@correo.facfor.unam.edu.ar

${ }^{2}$ Ing. Ftal. Profesional Independiente - Puerto Esperanza - Misiones
} 
que dependerán mucho del uso final de la materia prima.

Unos de los parámetros es la densidad básica, que caracteriza tecnológicamente la madera, presentando una acentuada correlación con la mayoría de las características físicas y mecánicas. Por otra parte se debe tener en cuenta en los programas de mejoramiento genético, medible con vista al mejor uso de las procedencias y más aún para la selección de individuos, dada la gran variación que a nivel individual presenta dicho carácter.

El conocimiento de la variación de las propiedades físicas y químicas del leño, que están relacionadas con la calidad y rendimiento en su futuro aprovechamiento, es esencial en los estudios de mejoramiento forestal.

Existe entre los investigadores del área tecnológica la preocupación en conocer las variaciones de las propiedades de las maderas producidas en las diversas especies de Pinus de rápido crecimiento, que ocurren cuando se consideran distintos tipos de suelo, edades, sistema de plantación, tratamientos silviculturales, procedencias, etc.

\section{OBJETIVOS}

- Evaluar las plantaciones de Pinus taeda a través de la densidad básica media de los árboles, cultivados en la Provincia de Misiones y norte de Corrientes, para diferentes edades y procedencias y el grado de variación que pueda existir.

- Determinar correlaciones entre la densidad básica obtenida con los tarugos y con las probetas extraídas de secciones transversales de las toras y confección de modelos matemáticos para la determinación de densidad de los árboles a distintas alturas.

\section{REVISIÓN BIBLIOGRÁFICA}

En la madera, como en cualquier cuerpo poroso se pueden indicar dos tipos de densidades:

a) La densidad real de la madera, que es aquella que corresponde al peso real del material leñoso, cuando se descuentan en la valoración del volumen todos los espacios huecos interiores incluidos los capilares; generalmente el valor de $1,5 \mathrm{~g} / \mathrm{cm}^{3}$ se considera como aceptable.
Según Noack (1964), la densidad real de la celulosa pura es de $1,58 \mathrm{~g} / \mathrm{cm}^{3}$, mientras que el de la lignina es de $1,40 \mathrm{~g} / \mathrm{cm}^{3}$. Por otra parte este autor determina que la resina en la madera tiene una densidad real de $0,90 \mathrm{~g} / \mathrm{cm} 3$, taninos $1,40 \mathrm{~g} / \mathrm{cm}^{3}$.

b) La densidad aparente de la madera, es decir la relación del peso al volumen de la muestra de madera sin descontar los espacios huecos internos.

La densidad constituye unas de las propiedades físicas de la madera, y de ella dependen la mayoría de sus características físicas y mecánicas, sirviendo en la práctica como referencia para clasificarlas, Coronel (1994).

La densidad posee además un patrón de variación con la edad y la altura, de tal manera que durante los primeros años el árbol produce madera con anillos de crecimiento anchos, donde hay una mayor proporción de madera temprana de baja densidad. Por otra parte respecto a la altura, la densidad es mayor en la base y va disminuyendo hacia la copa. Megraw, (1985).

La relación entre la densidad básica y las diversas propiedades de resistencia de la madera verde y seca demuestran que tales propiedades tienen el valor de resistencia mayores con un aumento de la densidad básica, notándose que esa relación es mayor para maderas secas que maderas verdes. La densidad básica es un excelente indicativo de las propiedades mecánicas en maderas de grano derecho y libres de defectos, o sea sin influencia de otros factores, que alteran negativamente sobre tal relacionamiento; Kollmann \& Cote Jr. (1968).

Knigge \& Schulz (1966), concuerdan en que maderas de coníferas con anillos de crecimiento más estrechos son más pesadas y resistentes, y ocurre lo contrario con las latifoliadas. Otros investigadores por su parte indican que el porcentaje de leño tardío influye de manera más directa en la densidad básica y consecuentemente en la resistencia cuando se lo compara con el ancho de los anillos de crecimiento.

Taras (1965), estudiando algunas propiedades de la madera de Pinus elliottii y su relación con la edad para cada tipo de leño producido por la especie, observó una 
disminución de la densidad básica del leño inicial con el aumento de la edad, equilibrándose a $\operatorname{los} 8$ a 12 años. Por otra parte, la densidad básica del leño tardío aumentó rápidamente en este período de crecimiento, equilibrándose en determinado momento $\mathrm{y}$ decreciendo en la dirección de la corteza. Sobre el total de los anillos de crecimiento (leño temprano y tardío), se pudo evidenciar un aumento de la densidad básica entre 8 a 12 años, luego se produce rápidamente un equilibrio.

La mayor variación de densidad ocurre dentro de un anillo de crecimiento anual, debido a que en el género Pinus presenta diferencias muy acentuadas entre el leño de primavera y el leño de otoño. Por ejemplo Ifju, (1969) citado por Zobel \& Van Buijtenen (1989), demostró que la densidad del leño de primavera de Pinos del Sur de Estados Unidos varía entre 0,30 a $0,46 \mathrm{~g} / \mathrm{cm}^{3}$, mientras que la densidad del leño de otoño varía entre 0,65 a $0,92 \mathrm{~g} / \mathrm{cm}^{3}$.

El Pinus taeda, como la mayoría de las coníferas, posee un patrón uniforme de baja densidad básica en la médula, un rápido aumento de la densidad en el período juvenil, seguido por series de anillos que poseen densidades esencialmente constantes, pero que fluctúan de año a año. Este patrón de variación para el Pinus taeda fue mostrado por diversos investigadores. Megraw, (1985) determinó que la densidad del Pinus taeda aumenta del centro hacia la parte externa en el sentido radial en toda la altura del árbol, pero que es mayor en la base que en la parte superior del árbol. Normalmente el cambio de densidad de la médula hacia la corteza, es mayor en el leño de otoño y menor en el leño de primavera Peterson, (1968).

Para poder comprender mejor los cambios de densidad de la madera con la altura dentro del árbol, se hace necesario estudiar las tendencias de la madera juvenil y la madera adulta separadamente. Megraw (1985), realizó un estudio, encontrando una fuerte disminución de la densidad de la madera juvenil desde la base del árbol hasta los $3 \mathrm{~m}$, por encima de los $5 \mathrm{~m}$ la densidad se mantuvo constante, independientemente de la altura.

Una de las variabilidades a tener en cuenta, es la variación de la densidad básica con la edad del árbol. Esta, está ligada también al porcentaje de madera juvenil y madera adulta. Los árboles más viejos poseen mayor densidad debido al mayor porcentaje de madera adulta y a la deposición de material resinoso en el cerne. Por otra parte aparece un fenómeno no muy común relatado por Talbert \& Jett (1981), donde la madera juvenil de los árboles más viejos de Pinus taeda poseen considerablemente mayor densidad que la madera juvenil de árboles más jóvenes. No existen este tipo de estudios para otras especies, pero para el Pinus taeda quedó muy claro y el aumento fue muy grande. Las razones son desconocidas, pero se cree que resultan de las tensiones del individuo, referido a una teoría de compactación.

La tasa de crecimiento es otro factor que tiene influencia en las propiedades de la madera. De acuerdo a Erikson \& Harrinson (1974), un aumento rápido de la tasa de crecimiento da como resultado variaciones en las propiedades, entre ellas un aumento en el porcentaje de leño inicial, reducción de la densidad básica y del largo de las traqueidas.

El conocimiento de la densidad básica es importante, ya que permite dar conclusiones sobre la adaptabilidad de la madera como material de construcción para fines estructurales, debido a la buena relación de resistencia/masa que presenta, Trendelenbur \& Mayer- Wegelin, (1956).

De acuerdo con los autores mencionados en el párrafo anterior, la gran variabilidad de madera se puede expresar por la variación de la densidad básica. Esta afirmación es corroborada por Bendtsen, (1978).

Según Kollmann (1951), en el caso específico de los pinos, las variaciones de la densidad básica pueden ser tan acentuadas en el sentido longitudinal del árbol al punto de sugerir diferentes clases de calidad solo en función de la posición del origen de las piezas.

\section{MATERIALES Y MÉTODOS}

El material de ensayo que se utilizó en el presente trabajo se obtuvo de tres áreas seleccionadas de plantaciones de la Provincia de Misiones y norte de la Provincia de Corrientes. En la tabla 1 se observan las procedencias, edades, alturas y diámetros de los árboles utilizados. 
Tabla 1: Procedencia, edad, región, y características de diámetro y altura de los árboles utilizados

Table 1: Procedure ag region, and caracteristics of diameter and height of the utilized trees

\begin{tabular}{|c|c|c|c|c|c|c|c|}
\hline Procedencia & Región & $\begin{array}{l}\text { Edad } \\
\text { (años) }\end{array}$ & $\begin{array}{l}\text { Diámetro máximo } \\
\text { (cm) }\end{array}$ & $\begin{array}{c}\text { Diámetro mínimo } \\
\text { (cm) }\end{array}$ & Altura máxima (m) & $\begin{array}{l}\text { Altura mínima } \\
\text { (m) }\end{array}$ & $\begin{array}{c}\text { Observa- } \\
\text { ciones }\end{array}$ \\
\hline Marion & $\begin{array}{l}\text { Norte de } \\
\text { Misiones. }\end{array}$ & 16 & 44,6 & 26,5 & 28 & 23,80 & Discos \\
\hline Marion & $\begin{array}{l}\text { Norte de } \\
\text { Misiones }\end{array}$ & 16 & & & & & Tarugos \\
\hline Local & $\begin{array}{l}\text { Centro de } \\
\text { Misiones. }\end{array}$ & 11 & 34,50 & 14,50 & 19,30 & 12,80 & Tarugos \\
\hline Local & " & 23 & 50,00 & 26,50 & 27,60 & 19,60 & $"$ \\
\hline Livingston & $\begin{array}{c}\text { Norte de } \\
\text { Corrientes. }\end{array}$ & 13 & 44,40 & 26,20 & 22,80 & 20,00 & $"$ \\
\hline Livingston & $"$ & 8 & 27,21 & 16,55 & 13,30 & 11,00 & $"$ \\
\hline Local & $"$ & 25 & 49,97 & 30,08 & 26,80 & 23,70 & $"$ \\
\hline Marion & $"$ & 12 & 33,42 & 19,09 & 19,10 & 16,20 & $"$ \\
\hline Livingston & $\begin{array}{l}\text { Norte de } \\
\text { Misiones. }\end{array}$ & 14 & 39,50 & 21,50 & 24,00 & 18,50 & $"$ \\
\hline Marion & $"$ & 6 & 23,50 & 12,00 & 12,00 & 8,00 & $"$ \\
\hline Marion & $"$ & 13 & 37,00 & 22,00 & 21,50 & 16,00 & $"$ \\
\hline Marion & $\begin{array}{l}\text { Centro de } \\
\text { Misiones. }\end{array}$ & 13 & 44,50 & 23,80 & 25,10 & 16,70 & $"$ \\
\hline Livingston & $"$ & 7 & 30,00 & 18,40 & 16,00 & 10,50 & $"$ \\
\hline Livingston & $"$ & 12 & 35,30 & 18,70 & 20,10 & 14,60 & $"$ \\
\hline Marion & $"$ & $6-7$ & 25,20 & 13,40 & 12,70 & 9,40 & $"$ \\
\hline Local & $\begin{array}{l}\text { Norte de } \\
\text { Misiones. }\end{array}$ & 24 & 55,38 & 32,14 & 27,70 & 22,30 & $"$ \\
\hline Local & $"$ & 14 & 39,00 & 20,90 & 20,50 & 16,20 & $"$ \\
\hline
\end{tabular}

Para la obtención del material, se seleccionaron plantaciones de tres procedencias de Pinus taeda (Marion, Livingston y Local), con un rango de edad de 6 a 23 años con una densidad inicial de plantación de $3 \mathrm{~m} \mathrm{x} \mathrm{1,5} \mathrm{m} \mathrm{(2.222} \mathrm{plantas} \mathrm{por}$ hectárea), y sustentadas sobre suelo rojo, Complejo 9, según, C.A.R.T.A. (1964)

La unidad de muestreo fue de 50 hectáreas, en las cuales se seleccionó 25 árboles libres de defectos, procediéndose con igual criterio en cada región, por procedencia y por edad. El error de muestreo fue de aproximadamente 5\% Prodepef, (1976).

\section{Metodología adoptada para determinar el punto de muestreo:}

En cada unidad de 50 hectáreas se escogieron al azar 5 centros de muestreo, procediéndose de la siguiente manera:

1. Fue seleccionado un extremo de la plantación al azar, a efectos de dar inicio a la tarea

2. Partiendo del extremo previamente seleccionado, se escogió un azimut al azar y luego a una distancia de 25 metros aproximadamente se obtuvo el primer punto de muestreo.

3. Este punto fue usado como partida para seleccionar el segundo azimut.

4. En el caso en que el punto de muestreo cayera fuera de la superficie de muestreo, por ser un azimut elegido al azar, ese punto fue eliminado, pero se siguió utilizando como referencia para el próximo.

5. De cada punto de muestreo fueron seleccionados cinco árboles, representativos de cada clase diamétrica, con un fuste único $\mathrm{y}$ recto $\mathrm{y}$ sin problemas fitosanitarios.

\section{Toma de muestra}

En la toma de muestra de los árboles seleccionados, se consideraron dos métodos que a continuación se describen:

\section{Método destructivo}

El material extraído por esta metodología (discos), fue utilizado en el análisis de la densidad en toda la altura de cada árbol.

Este material fue utilizado luego para realizar la correlación entre la densidad básica de los tarugos y las distintas alturas del árbol. 
De estos mismos árboles fueron extraídos tarugos, dos de cada individuo, al DAP.

En conjunto los discos y los tarugos de estos árboles corresponden a un primer grupo de muestreo, que fue utilizado a efectos de poder determinar un modelo estadístico que relacione la densidad básica de los tarugos con la densidad básica del árbol a distintas alturas.

Procedimiento: $\begin{array}{ccc}\text { El procedimiento consistió en apear los } \\ \text { árboles } & \text { seleccionados previamente, }\end{array}$ identificarlos y luego de cada árbol extraer discos (espesor de $2,5 \mathrm{~cm}$ ) a distintas alturas (desde la base hasta altura comercial: $0,30 \mathrm{~m} ; 1$ $\mathrm{m} ; 1,30 \mathrm{~m}$; y luego a cada 1,5 m). En la figura 1 , se puede observar, como fueron extraídos los discos, y se especifica la altura de la cuales fueron tomados.

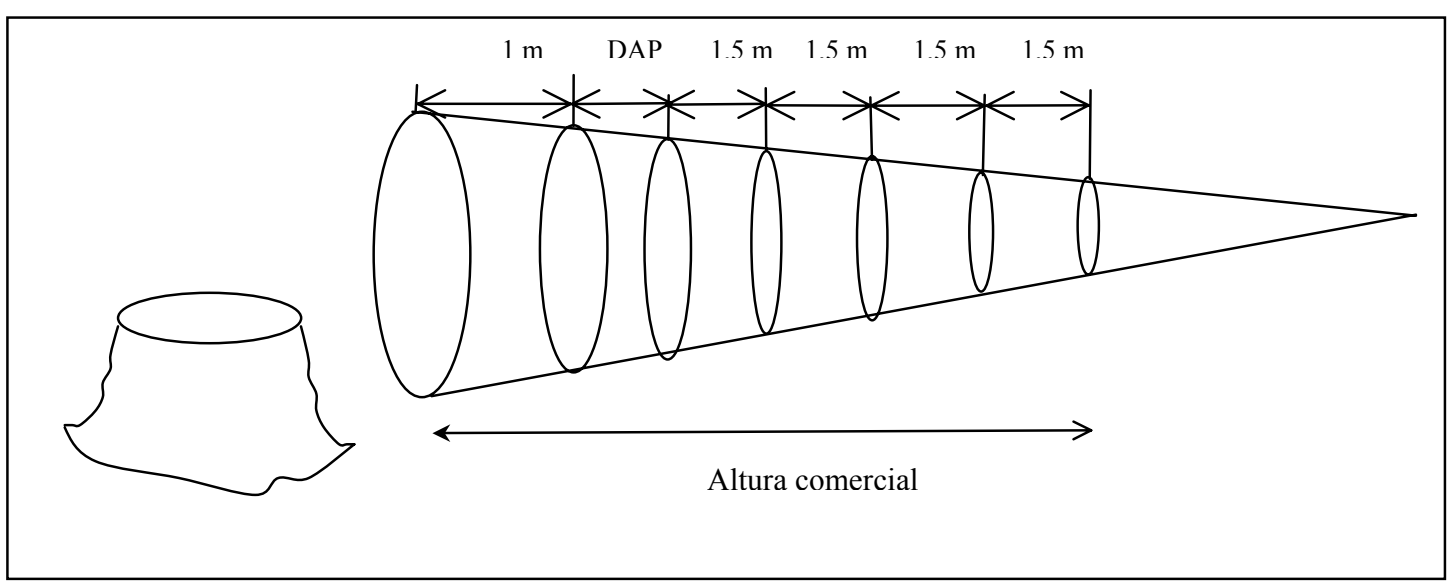

Figura 1: Posiciones de donde fueron extraídos los discos para la determinación de Densidad básica Figure 1: Posicion from where cross sections (disks) were stracted for basic density determination

\section{Método no destructivo}

De todos los árboles seleccionados como material de ensayo se extrajeron tarugos, utilizando para ello un Barreno de Pressler de $5 \mathrm{~mm}$ de diámetro.

Procedimiento:

Fueron extraídos de cada árbol y a altura de pecho (DAP: $1,30 \mathrm{~m}$ ) dos tarugos formando entre sí un ángulo de $90^{\circ}$, figura 2.
Después de tomar las muestras de los árboles con el barreno, se taparon los huecos con una madera del mismo diámetro para evitar la entrada de insectos u hongos y para facilitar la cicatrización de la herida más rápidamente. Los tarugos fueron cuidadosamente extraídos, buscando que dicho material siempre tenga como parte externa corteza y como extremo interno médula. El material fue correctamente identificado, en número de árbol y número de tarugo. 


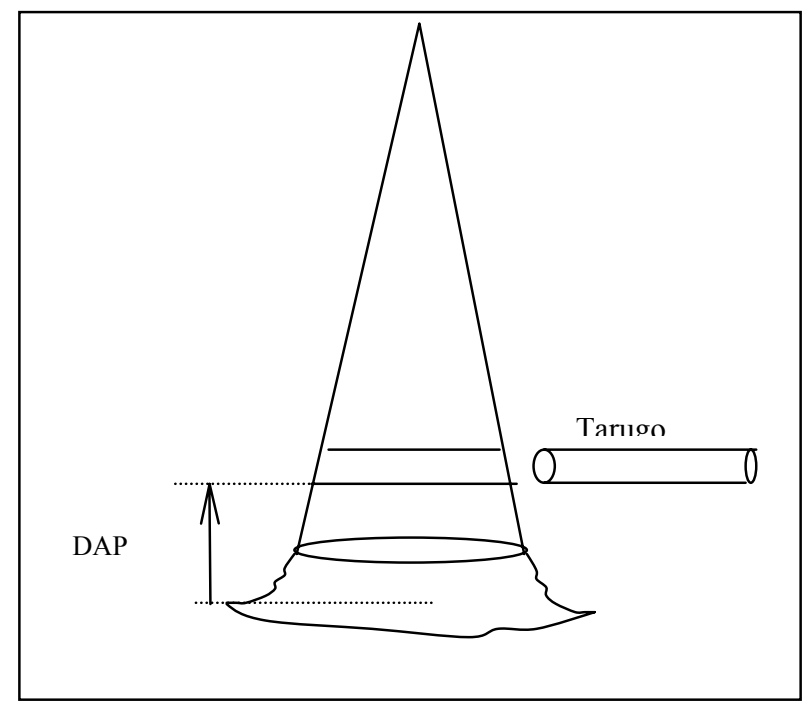

Figura 2: Posición de extracción del tarugo del árbol Figure 2: Increment corr stration position in the tree

\section{Metodología utilizada para determinar la densidad básica de los discos y los tarugos.}

Densidad básica de los discos

De cada disco traído del campo fueron tomadas dos cuñas representativas de todo el material. Cada cuña fue seccionada en tres partes (externa, media e interna) correspondiendo la externa al sector de corteza y la interna a la médula del material leñoso.

A efectos de tener seguridad de que no hubo pérdida de humedad en el material a ensayar, desde el momento de apeo y llegada al laboratorio, todo el material, antes de determinar el volumen fue sumergido en agua hasta saturación.

Una vez saturado, se tomó cada unas de las partes de la cuña y se determinó el peso saturado, para luego llevar a estufa hasta peso constante, a una temperatura de $103 \pm 2^{\circ} \mathrm{C}$; determinándose en este momento el peso seco de la muestra.

Con estos datos se determinaron los valores de peso saturado y peso anhidro de todo el material en estudio.

Para la determinación de la densidad básica, se utilizó el método del máximo tenor de humedad (Foelkel et al, 1971), que consiste en determinar el peso húmedo de la muestra y su respectivo peso absolutamente seco (Anhidro).

Los cálculos fueron realizados con ayuda de la siguiente fórmula:
$D b=$ densidad básica

$$
D b=\frac{P s-0,347}{P S}
$$

$P S=$ Peso saturado

$P s=$ Peso seco

Densidad básica de los tarugos.

El volumen de los tarugos para determinar la densidad básica fue realizada por el principio de Arquímedes, ya que se considera uno de los más prácticos y preciso.

Fue utilizado este método, debido a que los valores que arrojaba en trabajos de prueba, presentaron diferencias poco significativas respecto a otros métodos más precisos como la determinación del volumen utilizando el Volumenómetro de Breuil.

Se trata de medir el volumen de la probeta de madera en función del peso del líquido desplazado por inmersión, o sea por medición del empuje.

Al sumergir la pieza en un recipiente con agua, se produce una diferencia, equivalente al peso del agua desplazada, que equivale al volumen de la pieza sumergida.

Se puede establecer una ecuación de equilibrio:

$$
\mathrm{P}=\mathrm{E}
$$

Donde: 
$\mathrm{P}$ (peso del líquido desplazado) $\mathrm{g}$

E (empuje del líquido desplazado) g

Por hidrostática se tiene:

$$
\mathrm{E}=\mathrm{V} \cdot \mathrm{d}
$$

Donde:

$\mathrm{V}=$ (volumen del liquido) $\mathrm{cm}^{3}$

$\mathrm{d}=$ (densidad del líquido) $\mathrm{g} / \mathrm{cm}^{3}$

Entonces: $\mathrm{P}=\mathrm{V} . \mathrm{d}$, de donde se tendrá que, $\mathrm{V}$ $=\mathrm{P}\left(\mathrm{cm}^{3}\right)$

\section{RESULTADOS Y DISCUSIÓN}

\section{Análisis y discusión de la Densidad básica obtenido de los tarugos, de las distintas regiones, procedencias y clases de edad.}

Al comenzar este estudio se pensó en recopilar material abarcando todas las edades, procedencias y regiones, pero se presentaron dificultades de aspectos diversos imposibilitando reunirse con el material deseado y abarcar todas las procedencias, regiones y edades.

Por ello se entregan resultados obtenidos de las densidades de los tarugos de las 16 áreas muestreadas.

Los resultados presentados en la tabla 2 , corresponden a los obtenidos a través del método no destructivo (tarugos), los mismos fueron extraídos al DAP, según se indica en la metodología del presente trabajo, y en la misma se puede observar el comportamiento de las tres regiones, las tres procedencias y las cuatro clases de edades analizadas.

Tabla 2: Densidades básicas medias de tarugos, de las combinaciones procedencia - clase de edad - área Table 2: Average basic density from cores, of the combinations procedure - age class - area

\begin{tabular}{|c|c|c|c|c|}
\hline Combinaciones & $\begin{array}{c}\text { Sección externa } \\
\text { Densidad básica } \\
\left(\mathbf{g} / \mathbf{c m}^{3}\right)\end{array}$ & $\begin{array}{c}\text { Sección media } \\
\text { Densidad básica } \\
\left(\mathrm{g} / \mathrm{cm}^{\mathbf{3}}\right)\end{array}$ & $\begin{array}{c}\text { Sección interna } \\
\text { Densidad básica } \\
\left(\mathrm{g} / \mathrm{cm}^{3}\right)\end{array}$ & $\begin{array}{c}\text { Media general } \\
\text { Densidad básica } \\
\left(\mathrm{g} / \mathrm{cm}^{3}\right)\end{array}$ \\
\hline $\mathrm{LO}-4$ - NM & 0,601 & 0,481 & 0,384 & 0,488 \\
\hline LO - 4 - CM & 0,596 & 0,458 & 0,378 & 0,477 \\
\hline $\mathrm{LO}-4-\mathrm{NC}$ & 0,570 & 0,460 & 0,411 & 0,480 \\
\hline $\mathrm{LO}-2-\mathrm{CM}$ & 0,533 & 0,429 & 0,388 & 0,433 \\
\hline LO - 2 - NM & 0,513 & 0,371 & 0,319 & 0,401 \\
\hline MA - 3 - NM & 0,540 & 0,400 & 0,346 & 0,428 \\
\hline $\mathrm{MA}-2$ - NC & 0,493 & 0,384 & 0,335 & 0,404 \\
\hline $\mathrm{MA}-2$ - CM & 0,468 & 0,375 & 0,339 & 0,394 \\
\hline LI - 2 - NM & 0,567 & 0,378 & 0,360 & 0,435 \\
\hline LI - 2 - CM & 0,510 & 0,416 & 0,351 & 0,425 \\
\hline $\mathrm{LI}-2$ - NC & 0,485 & 0,395 & 0,348 & 0,410 \\
\hline $\mathrm{LI}-1-\mathrm{NC}$ & 0,441 & 0,400 & 0,331 & 0,391 \\
\hline LI - 1 - CM & 0,424 & 0,386 & 0,333 & 0,381 \\
\hline MA - 1 - NM & 0,380 & 0,375 & 0,339 & 0,364 \\
\hline $\mathrm{MA}-1-\mathrm{CM}$ & 0,373 & 0,342 & 0,325 & 0,346 \\
\hline MA - 2 - NM & 0,568 & 0,404 & 0,351 & 0,441 \\
\hline
\end{tabular}

\begin{tabular}{|c|c|}
\hline Clase de edad & Años \\
\hline 1 & 6 a 10 \\
\hline 2 & 11 a 15 \\
\hline 3 & 16 a 20 \\
\hline 4 & 21 a 25 \\
\hline
\end{tabular}

LO: Procedencia Local

MA: Procedencia Marion LI: $\quad$ Procedencia Livingston
NM: $\quad$ Norte de Misiones

CM: Centro de Misiones

NC: $\quad$ Norte de Corrientes
De los resultados obtenidos, se observa que tomando la clase de edad 4, para una misma procedencia (local), las densidades no son significativamente diferentes, observándose una densidad mayor para la región Norte de Misiones, seguida por el Centro de Misiones y luego el Norte de 
Corrientes. $\quad\left(0,601 \mathrm{~g} / \mathrm{cm}^{3}-\quad 0,596 \mathrm{~g} / \mathrm{cm}^{3} \quad\right.$ $0,570 \mathrm{~g} / \mathrm{cm}^{3}$ respectivamente).

Para el caso de la clase de edad 2, para la misma procedencias, no existe diferencias entre las regiones Centro de Misiones y Norte de Misiones, arrojando los siguientes valores medios $\left(0,533 \mathrm{~g} / \mathrm{cm}^{3}\right.$ y $\left.0,513 \mathrm{~g} / \mathrm{cm}^{3}\right)$.

Analizada la clase diamétrica 2, para la procedencia Marion, se observa que existe una mayor densidad básica en la región norte de Misiones cuando se compara con el Norte de Corrientes y centro de Misiones $\left(0,568 \mathrm{~g} / \mathrm{cm}^{3}\right.$ $\left.0,493 \mathrm{~g} / \mathrm{cm}^{3}-0,468 \mathrm{~g} / \mathrm{cm}^{3}\right)$. La clase de edad 1 , presenta una mayor densidad en la región Norte de Misiones, comparado con el centro de Misiones $\left(0,380 \mathrm{~g} / \mathrm{cm}^{3}-0,373 \mathrm{~g} / \mathrm{cm}^{3}\right)$.

$\mathrm{La}$ procedencia Livingston, clase diamétrica 2 , presenta una mayor densidad básica en la región Norte de Misiones, seguida por el Centro de Misiones y luego el Norte de Corrientes $\quad\left(0,567 \mathrm{~g} / \mathrm{cm}^{3}-0,510 \mathrm{~g} / \mathrm{cm}^{3}\right.$ $\left.0,485 \mathrm{~g} / \mathrm{cm}^{3}\right)$. La clase de 1 , presenta una densidad básica mayor en la región Norte de Corrientes comparado con el Centro de Misiones, $\left(0,441 \mathrm{~g} / \mathrm{cm}^{3}-0,424 \mathrm{~g} / \mathrm{cm}^{3}\right)$.

Cuando se analizan en forma conjunta, se observa que la densidad de la madera aumenta con la edad, independientemente de la procedencia o región. O sea que la densidad de la madera depende casi exclusivamente de la proporción de leño tardío y leño temprano. Esta proporción aumenta acorde a la edad del árbol.

En un rango de edades entre 6 a 10 años, se observa que la Densidad Básica es mayor para Livingston que para Marion. Se piensa que la diferencia no es sustancialmente mayor debido que en Pinus taeda la formación de madera madura se produce a partir del décimo año, y ésta es la que define o marca la diferencia.

Es de destacar que para edades mayores entre 11 a 15 años, la madera de procedencia Livingston es más densa que la de procedencia Marion.

Para todas las procedencias, y para las edades que se consideran, la densidad de la madera tiene una declinación de Norte a Sur.

No se puede explicar porque la densidad básica de la procedencia Marion para una edad de 15 años es mayor que para 16 años, tanto en su valor promedio como para cada una de sus porciones.
Análisis y discusión de los modelos obtenidos para relacionar la densidad básica de las cuñas a distintas alturas del árbol sobre base de la densidad básica de los tarugos al DAP.

Analisis de regresion en modelos de prediccion de la densidad basica del leño a distintas alturas de fuste

- Selección de modelos

En primer lugar se analizaron modelos lineales de por lo menos dos variables independientes, teniendo en cuenta la densidad básica (DB) de los tarugos y la altura de las cuñas en las que se registraron las observaciones de densidad.

En cada caso se obtuvo el cuadro resumen de cada regresión efectuada, dando especial interés al coeficiente de determinación $\mathrm{R}^{2}$, (ajustado y sin ajustar), a los resultados de la prueba $\mathrm{F}$ para el modelo, a los coeficientes de regresión estimados y el nivel de significación de cada uno de ellos. En los primeros modelos analizados se llevaron a cabo los análisis de residuales.

Luego se realizaron pruebas con cada modelo ajustado dentro del rango de variación de cada variable de respuesta: densidad básica externa de la cuña (DBECU); densidad básica media de la cuña (DBMCU) y densidad básica interna de la cuña (DBICU) para observar el comportamiento de cada modelo en la predicción de la densidad básica (DB) a distintas alturas de fuste según la densidad básica (DB) correspondiente de los tarugos.

- Modelos de regresión para la densidad básica externa de las cuñas (DBECU)

Para llegar al modelo final se realizaron análisis de varios modelos lineales, el primero fue realizado para la parte externa de la cuña, se inició el proceso con:

$\mathrm{DBECU}=\mathrm{B} 0+\mathrm{B} 1 * \mathrm{DBET}+\mathrm{B} 2 * \mathrm{HC}+$ error;

Con los siguientes resultados del ajuste y una prueba de funcionamiento en la predicción dados los valores de altura de cuña (HC) y densidad básica externa de los tarugos (DBET) elegidos arbitrariamente, tabla 3. 
Tabla 3: Resumen de regresión efectuada para determinar el modelo para la DBECU, donde se observan resultados de la prueba $\mathrm{F}$ para el modelo, los coeficientes de regresión estimados y el nivel de significación para cada uno de ellos

Table 3: Coefficients and statistics regression summary for dependent variable: DBECU

\begin{tabular}{l}
\hline $\begin{array}{l}\text { Regression Summary for Dependent Variable: DBECU } \\
\mathrm{R}=, 87789942 \\
\mathrm{R}\end{array} \mathrm{R}=, 77070740$ Adjusted $\mathrm{R}^{2}=, 76935064$ \\
$\mathrm{~F}(2,338)=568,05 \mathrm{p}<0,0000$ Std.Error of estimate:, 03631 \\
\hline
\end{tabular}

Análisis de residuos

Fueron realizados los análisis residuales para los modelos lineales estudiados.

En el gráfico 1 se observa una leve tendencia cuadrática de los residuos estandarizados respecto de la altura de cuña, lo cual lleva a explorar sobre un modelo que contenga un término de altura de cuña (HC) al cuadrado.

En el gráfico 2, se observa un valor raro que se debe investigar y una leve presencia de heterocedasticidad, lo cual lleva a plantear un modelo para la densidad básica externa de la cuña (DBECU) que contenga alguna transformación de densidad básica externa de tarugos (DBET), por ejemplo una transformación logarítmica.

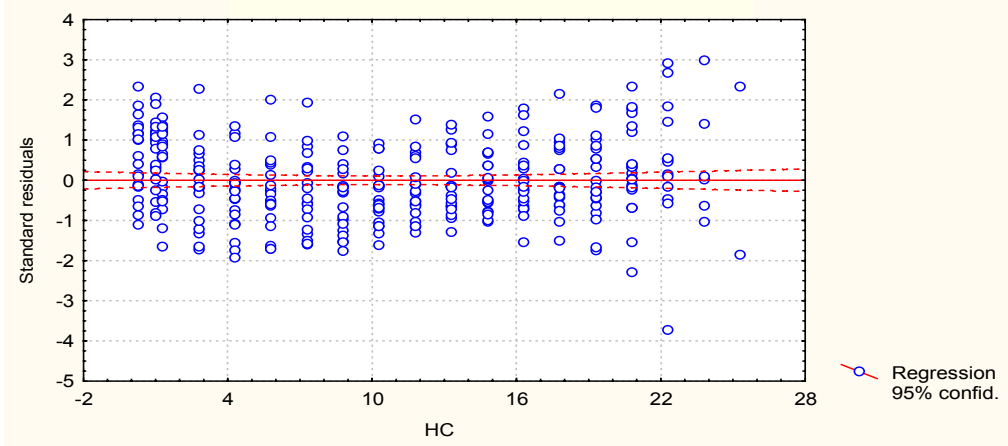

Grafico 1: Residuos estandarizados vs. altura de cuña (HC)

Figure 1: Standaized residuals vs. edge height (HC) 


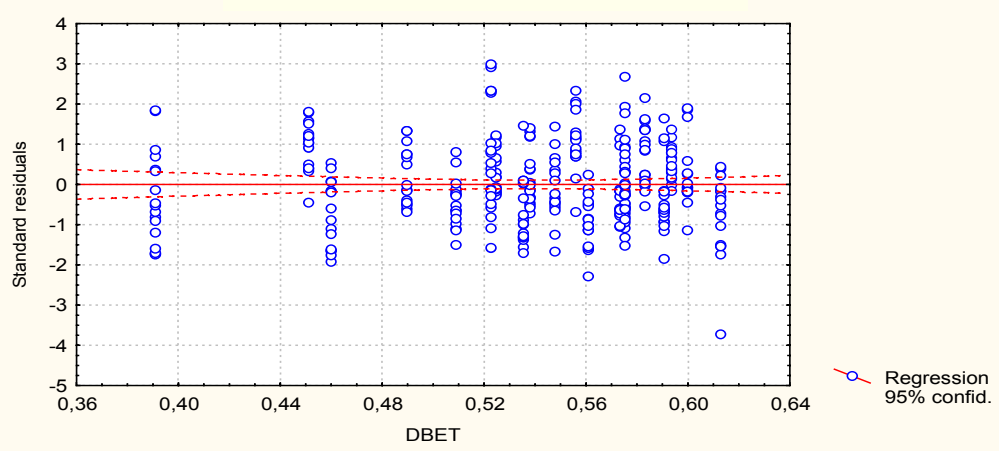

Grafico 2: Residuos estandarizados vs. densidad básica externa de los tarugos (DBET)

Figure 2: $\quad$ Standaized residuals vs extern basic density of the cores (DBET)

Ajustados los modelos con altura de cuña (HC) al cuadrado y/o ln de la densidad básica externa de los tarugos (DBET), no se obtuvieron mejores indicadores de ajuste $\mathrm{R} o$ $\mathrm{R}^{2}$, ni tampoco mejores funcionamientos de los modelos para predecir la densidad básica externa de las cuñas (DBECU) con sentido biológico en alturas por debajo de los $2 \mathrm{~m}$ y la densidad básica externa de los tarugos (DBET) de 0,6 .

Eliminado un valor aberrante cuyo residual estandarizado superó al valor absoluto de 3, tampoco se mejoró la calidad del ajuste ni la capacidad de predicción de la densidad básica externa de la cuña (DBECU).

Análisis de superficie de respuesta

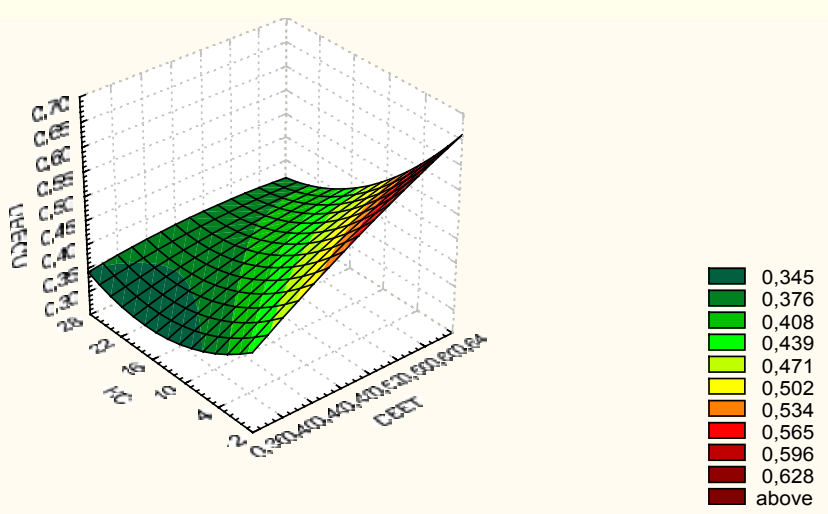

Grafico 3: Superficie de respuesta cuadrática suavizada para la densidad básica externa de la cuña (DBECU) Figure 3: $\quad$ Quadratic answer surface harmonized for the extern basic density of the edge (DBECU) 
En el grafico 3, se observa un aumento de la densidad básica externa (DBE) con el aumento de la densidad básica externa de los tarugos (DBET) y una disminución con el aumento de la altura de la cuña (HC).
El modelo completo con las dos variables regresoras con términos lineal $\mathrm{y}$ cuadrático más las interacciones produjo los siguientes resultados:

Tabla 4: Resumen de regresión efectuada para determinar el modelo de regresión para la DBECU, donde se observan resultados de la prueba $\mathrm{F}$ para el modelo, los coeficientes de regresión estimados y el nivel de significación para cada uno de ellos.

Table 4: Coefficients and statistics regression summary for dependent variable: DBECU

\begin{tabular}{|c|c|c|c|c|c|}
\hline \multicolumn{6}{|c|}{ Regression Summary for Dependent Variable: DBECU } \\
\hline \multicolumn{6}{|c|}{$\mathrm{R}=, 90446636 \mathrm{R}^{2}=0,81805939$ Adjusted $\mathrm{R}^{2}=0,81533573$} \\
\hline \multicolumn{6}{|c|}{$\mathrm{F}(5,334)=300,35 \mathrm{p}<0,0000$ Std.Error of estimate: 0,03221} \\
\hline & & & St. Err. & & \\
\hline & & B & of B & $\mathrm{t}(334)$ & p-level \\
\hline Intercpt & B0 & $-0,051$ & 0,126 & $-0,404$ & 0,686 \\
\hline DBET & B1 & 1,475 & 0,487 & 3,027 & 0,003 \\
\hline $\mathrm{HC} 2$ & $\mathrm{~B} 2$ & 0,000 & 0,000 & 6,715 & 0,000 \\
\hline DBET2 & B3 & $-0,655$ & 0,473 & $-1,384$ & 0,167 \\
\hline $\mathrm{HC}$ & B4 & 0,000 & 0,003 & 0,133 & 0,894 \\
\hline HC*DBET & B5 & $-0,027$ & 0,005 & $-5,856$ & 0,000 \\
\hline
\end{tabular}

Los coeficientes de determinación ( R2 y R2 ajustado) son levemente superiores a los de la tabla 3, las predicciones de la densidad básica externa (DBE) a bajas alturas son biológicamente más cercanas de la realidad, con la desventaja que hay tres estimaciones de coeficientes (b0, b3 y b4) que no son significativos aplicando la prueba $t$.

Se ajustó un modelo derivado del modelo completo sin considerar los términos cuyos coeficientes resultaron sin significación estadística para explicar el comportamiento de la variable respuesta. Los resultados se muestran en la tabla 5 .

Tabla 5. Resumen de regresión efectuada para determinar el modelo para la DBECU, donde se observan resultados de la prueba $\mathrm{F}$ para el modelo, los coeficientes de regresión estimados y el nivel de significación para cada uno de ellos.

Table 5: Coefficients and statistics regression summary for dependent variable: DBECU

\begin{tabular}{|c|c|c|c|c|c|}
\hline \multicolumn{6}{|c|}{ Regression Summary for Dependent Variable: DBECU } \\
\hline \multicolumn{6}{|c|}{$\mathrm{R}=, 90388532 \mathrm{R}^{2}=, 81700868$ Adjusted $\mathrm{R}^{2}=, 81537483$} \\
\hline \multicolumn{6}{|c|}{$F(3,336)=500,05 p<0,0000$ Std.Error of estimate:, 03221} \\
\hline & & & St. Err. & & \\
\hline & & $\mathrm{B}$ & of B & $\mathrm{t}(336)$ & p-level \\
\hline Intercpt & B0 & 0,1207 & 0,018 & 6,653 & 0,000 \\
\hline DBET & B1 & 0,8000 & 0,036 & 22,512 & 0,000 \\
\hline $\mathrm{HC}^{2}$ & $\mathrm{~B} 2$ & 0,0003 & 0,000 & 7,056 & 0,000 \\
\hline HC*DBET & B3 & $-0,0262$ & 0,002 & $-16,548$ & 0,000 \\
\hline
\end{tabular}

El modelo

$$
\mathrm{DBECU}=\mathrm{B} 0+\mathrm{B} 1 * \mathrm{DBET}+\mathrm{B} 2 * \mathrm{HC}^{2}+\mathrm{B} 3 *(\mathrm{HC} * \mathrm{DBET})+\text { error }
$$

Resultó ser el de mejor ajuste dentro de los modelos lineales ajustados, tiene un comportamiento más satisfactorio para predecir la densidad básica externa (DBE) a bajas alturas de fuste y todas las estimaciones de coeficientes son estadísticamente significativas usando la prueba t. Además es relativamente simple de usar, ya que no requiere transformaciones de variables y tiene sólo 4 términos a los efectos de producir 
predicciones de datos independientes de los que se usaron en el ajuste del modelo.

El valor predicho de densidad básica externa (DBE) a una dada altura de cuña (HC) (altura de fuste a la cual se quiere predecir la
DBE) una vez que se cuenta con el dato correcto de densidad básica externa del tarugo (DBET) en las unidades usadas en la toma de datos y $\mathrm{HC}$ en metros, será:

\section{DBECU $=0,1207+0,8000 *$ DBET $+0,0003 * H^{2}-0,0262 *(H C * D B E T)$}

Una prueba del funcionamiento del modelo se muestra en la Tabla 5. En la misma se puede observar el buen ajuste del modelo.

Tabla 6: Prueba de funcionamiento del modelo

Table 6: Prove of the model workness

\begin{tabular}{|c|c|c|c|}
\hline \multirow{2}{*}{ HC } & \multicolumn{3}{|c|}{ DBET } \\
\cline { 2 - 4 } & $\mathbf{0 , 4}$ & $\mathbf{0 , 5}$ & $\mathbf{0 , 6}$ \\
\hline 0,3 & 0,438 & 0,517 & 0,596 \\
\hline 2 & 0,421 & 0,496 & 0,571 \\
\hline 14 & 0,353 & 0,396 & 0,440 \\
\hline 20 & 0,351 & 0,379 & 0,407 \\
\hline
\end{tabular}

Los ajustes de modelos no lineales probados no tienen mejores coeficientes de determinación que los de la tabla 5 y además no resultaron eficientes para predicciones de la densidad básica externa (DBE).
Modelos de regresión para la Densidad básica media de la cuña. (DBMCU)

Con el modelo ya ajustado para la densidad básica externa de la cuña, se optó por ajustar directamente el modelo completo del tipo:

$\mathrm{DBMCU}=\mathrm{B} 0+\mathrm{B} 1 * \mathrm{DBMT}+\mathrm{B} 2 *(\mathrm{HC} * \mathrm{DBMT})+\mathrm{B} 3 * \mathrm{DBMT} 2+\mathrm{B} 4 * \mathrm{HC} 2+\mathrm{B} 5 * \mathrm{HC}+$ error

Tabla 7: Resumen de regresión efectuada para determinar la DBMCU, donde se observan resultados de la prueba $\mathrm{F}$ para el modelo, los coeficientes de regresión estimados y el nivel de significación para cada uno de ellos

Table 7: Coefficients and statistics regression summary for dependent variable: DBECU

\begin{tabular}{|c|c|c|c|c|}
\hline \multicolumn{5}{|c|}{ Regression Summary for Dependent Variable: DBMCU } \\
\hline \multicolumn{5}{|c|}{$\mathrm{R}=, 65519712 \mathrm{R}^{2}=, 42928326$ Adjusted $\mathrm{R}^{2}=, 42073960$} \\
\hline \multicolumn{5}{|c|}{$\mathrm{F}(5,334)=50,246 \mathrm{p}<, 00000$ Std.Error of estimate:, 03535} \\
\hline & & St. Err. & & \\
\hline & $\mathrm{B}$ & of B & $\mathrm{t}(334)$ & p-level \\
\hline Intercpt & $-0,388$ & 0,175 & $-2,215$ & 0,027 \\
\hline DBMT & 3,429 & 0,869 & 3,946 & 0,000 \\
\hline HC*DBMT & $-0,018$ & 0,007 & $-2,694$ & 0,007 \\
\hline DBMT2 & $-3,425$ & 1,077 & $-3,181$ & 0,002 \\
\hline $\mathrm{HC} 2$ & 0,000 & 0,000 & 4,166 & 0,000 \\
\hline $\mathrm{HC}$ & 0,000 & 0,003 & 0,141 & 0,888 \\
\hline
\end{tabular}

Analizando los residuales estandarizados se encontraron 4 valores raros $\mathrm{u}$ "outliers", los que quitados del análisis llevaron a un ajuste levemente superior al mostrado en la tabla 7 , con un coeficiente de determinación ajustado de 0.468 .
El ajuste de un modelo sin altura de cuña (HC), debido a que su coeficiente no es significativo para explicar DBMCU según la prueba $t$, y sin los outliers arrojó los siguientes resultados: 
Tabla 8: Resumen de regresión efectuada para determinar la DBMCU, donde se observan resultados de la prueba $\mathrm{F}$ para el modelo, los coeficientes de regresión estimados y el nivel de significación para cada uno de ellos

Table 8 Coefficients and statistics regression summary for dependent variable: DBECU

\begin{tabular}{|c|c|c|c|c|}
\hline Regression S & ry for D & Variab & & \\
\hline $\mathrm{R}=, 6887961$ &, 474440 & ted $\mathrm{R}^{2}=$ & & \\
\hline $\mathrm{F}(4,331)=74$ & $<, 00000$ & r of estil & 10 & \\
\hline & & St. Err. & & \\
\hline & $\mathrm{B}$ & of B & $\mathrm{T}(331)$ & p-level \\
\hline Intercpt & $-0,5132$ & 0,163 & $-3,142$ & 0,002 \\
\hline DBMT & 4,0309 & 0,816 & 4,937 & 0,000 \\
\hline HC*DBMT & $-0,0182$ & 0,002 & $-8,164$ & 0,000 \\
\hline DBMT2 & $-4,1373$ & 1,013 & $-4,083$ & 0,000 \\
\hline $\mathrm{HC} 2$ & 0,0002 & 0,000 & 5,156 & 0,000 \\
\hline
\end{tabular}

Los valores de los coeficientes de determinación son relativamente bajos, por lo que se hicieron algunas de las verificaciones a efectos de detectar alguna fuente de error de los registros.

Al no detectarse errores y ser sin embargo, todos los coeficientes estimados en el ajuste estadísticamente significativos se continúo con la búsqueda del modelo.

La prueba que consta en la tabla 9 muestra algunas inconsistencias casi insignificantes de las predicciones en valores de la densidad básica media del tarugo $(\mathrm{DBMT})=0.5$ y la densidad básica media del tarugo $(\mathrm{DBMT})=0.3$ (valores extremos), lo cual se puede apreciar en el gráfico 4.

Tabla 9: Prueba de funcionamiento del modelo

Table 9: $\quad$ Prove of the model workness

\begin{tabular}{|c|c|c|c|}
\hline PRUEBA & \multicolumn{3}{|c|}{ DBMT } \\
\hline HC & $\mathbf{0 , 3}$ & $\mathbf{0 , 4}$ & $\mathbf{0 , 5}$ \\
\cline { 2 - 4 } 0,3 & 0,322 & 0,435 & 0,466 \\
\hline 2 & 0,314 & 0,424 & 0,451 \\
\hline 14 & 0,288 & 0,376 & 0,381 \\
\hline 20 & 0,296 & 0,373 & 0,368 \\
\hline
\end{tabular}

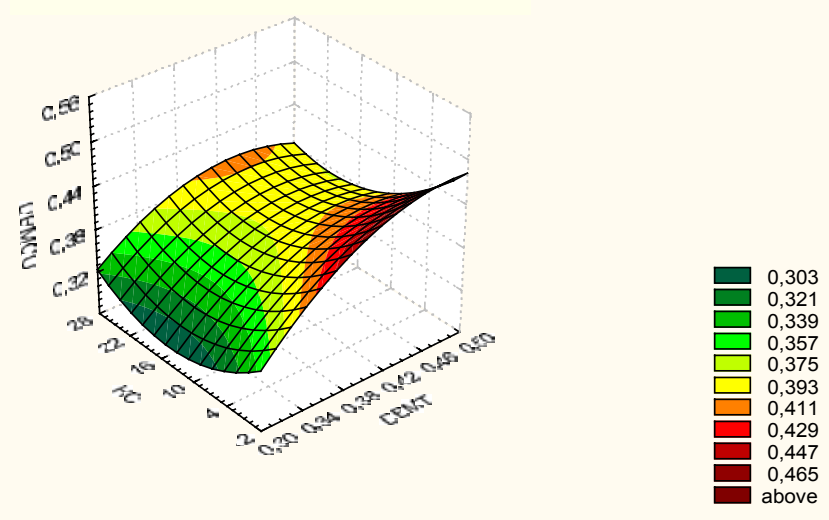

Gráfico 4: Superficie de respuesta cuadrática suavizada para la densidad básica media de la cuña (DBMCU)

Figure 4: Quadratic answer surface harmonized for the average basic density of the edge (DBECU) 
Con todas las restricciones del caso, se propone un modelo predictor de la siguiente forma:

Modelos de regresión para la Densidad básica interna de la cuña (DBICU)

DBMCU $=-0,513+4,031 *$ DBMT $-0,018 *\left(H^{*} *\right.$ DBMT $)-4,137 *$ DBMT $^{2}+0,0002 * \mathrm{HC}^{2}$

Se usó una metodología similar a la utilizada en la densidad básica media de la cuña (DBMCU), con los siguientes resultados:

Tabla 10: Resultados del ajuste del modelo "completo" para la densidad básica interna de las cuñas (DBICU)

Table 10: Results of the complet model fitting for intern basic density of the edges (DBICU)

\begin{tabular}{|c|c|c|c|c|}
\hline \multicolumn{5}{|c|}{ Regression Summary for Dependent Variable: DBICU } \\
\hline \multicolumn{5}{|c|}{$\mathrm{R}=, 61884611 \mathrm{R}^{2}=, 38297051$ Adjusted $\mathrm{R}^{2}=, 37373354$} \\
\hline \multicolumn{5}{|c|}{$\mathrm{F}(5,334)=41,461 \mathrm{p}<, 00000$ Std.Error of estimate:, 03203} \\
\hline & & St. Err. & & \\
\hline & B & of $B$ & $\mathrm{t}(334)$ & p-level \\
\hline Intercpt & 0,155 & 0,131 & 1,181 & 0,238 \\
\hline DBIT & 0,551 & 0,773 & 0,713 & 0,476 \\
\hline HC*DBIT & $-0,020$ & 0,008 & $-2,683$ & 0,008 \\
\hline DBIT2 & 0,171 & 1,147 & 0,149 & 0,882 \\
\hline $\mathrm{HC} 2$ & 0,000 & 0,000 & $-1,593$ & 0,112 \\
\hline $\mathrm{HC}$ & 0,006 & 0,003 & 2,061 & 0,040 \\
\hline
\end{tabular}

Luego de un proceso de selección los coeficientes estimados, se llegó a un teniendo en cuenta los coeficientes de modelo reducido con los siguientes resultados: determinación (ninguno superó el $\mathrm{R}^{2}$ ajustado de la tabla 11) y la significancia estadística de

Tabla 11: Resultados del ajuste del modelo reducido para la densidad básica interna de las cuñas (DBICU) Table 11: Results of the reduced model fitting for intern basic density of the edges (DBICU)

\begin{tabular}{|c|c|c|c|c|}
\hline \multicolumn{5}{|c|}{ Regression Summary for Dependent Variable: DBICU } \\
\hline \multicolumn{5}{|c|}{$\mathrm{R}=, 61191416 \mathrm{R}^{2}=, 37443894$ Adjusted $\mathrm{R}^{2}=, 37069307$} \\
\hline \multicolumn{5}{|c|}{$\mathrm{F}(2,334)=99,960 \mathrm{p}<, 00000$ Std.Error of estimate:, 03065} \\
\hline & & St. Err. & & \\
\hline & $\mathrm{B}$ & of $\mathrm{B}$ & $t(334)$ & $\overline{p-l e v e l}$ \\
\hline Intercpt & 0,182 & 0,018 & 10,135 & 0,000 \\
\hline DBIT & 0,540 & 0,052 & 10,302 & 0,000 \\
\hline HC*DBIT & $-0,008$ & 0,001 & $-11,069$ & 0,000 \\
\hline
\end{tabular}

Sobre este modelo que tiene la expresión

$$
\text { DBICU }=\text { B0 + B1 * DBIT + B2 * }(\text { HC*DBIT })+\text { error; }
$$

Se efectuó una prueba con los siguientes resultados, que muestran inconsistencia de las predicciones en valores bajos de la Densidad básica interna del tarugo (DBIT) dentro del rango de dispersión de los datos, tabla 12.

Tabla 12: Prueba de funcionamiento del modelo

Table 12: Prove of the model workness

\begin{tabular}{|c|c|c|c|}
\hline PRUEBA & \multicolumn{3}{|c|}{ DBIT } \\
\hline HC & $\mathbf{0 , 2 6}$ & $\mathbf{0 , 3 2}$ & $\mathbf{0 , 3 8}$ \\
\cline { 2 - 4 } 0,3 & 0,322 & 0,354 & 0,386 \\
\hline 2 & 0,318 & 0,350 & 0,381 \\
\hline 14 & 0,293 & 0,319 & 0,345 \\
\hline 20 & 0,281 & 0,304 & 0,326 \\
\hline
\end{tabular}


La observación anterior coincide parcialmente con la superficie de respuesta que muestra el gráfico 5.

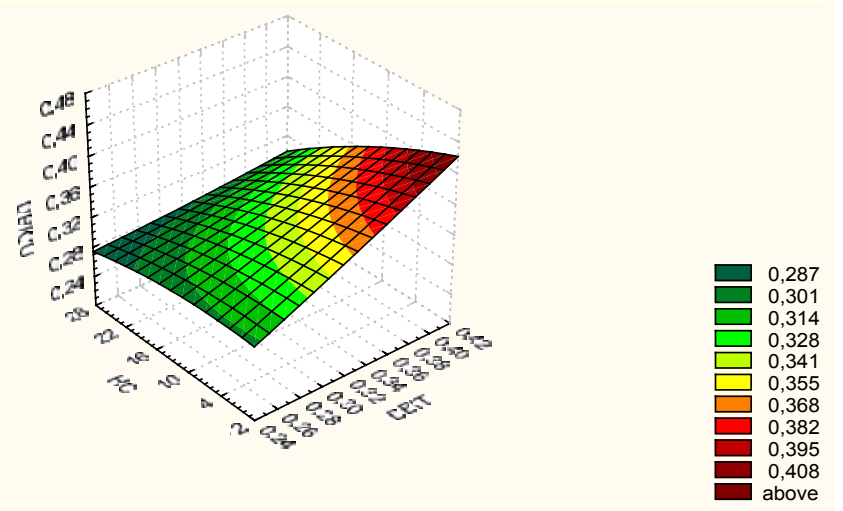

Gráfico 5: Superficie de respuesta cuadrática suavizada para la densidad básica interna de la cuña (DBICU)

Figure 5: Quadratic answer surface harmonized for the intern basic density of the edge (DBICU)

El gráfico 6 muestra indicios del comportamiento de las variables a través de una superficie de respuesta por mínimos cuadrados ponderados.

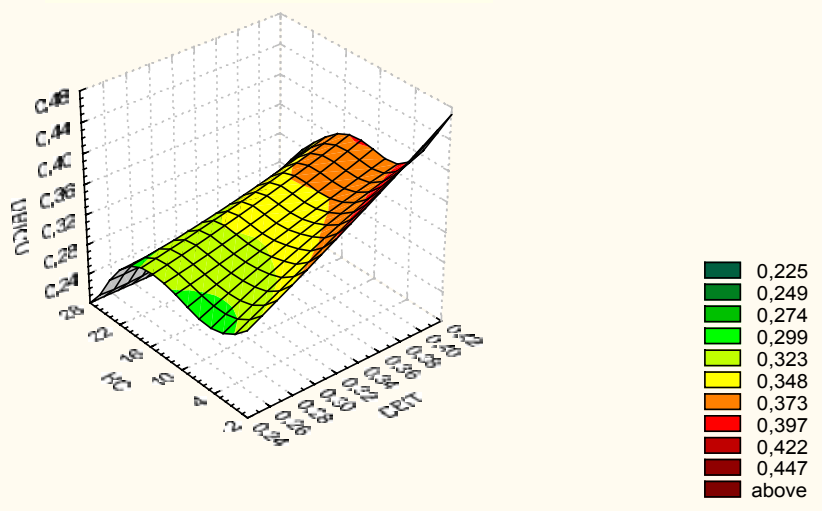

Gráfico 6: Superficie de respuesta de distancias mínimas cuadráticas ponderadas para DBICU

Figure 6: Answer surface of weighted quadratic minimum distances for DBICU

Se ajustaron 7 modelos no lineales con altura de cuña (HC) y densidad básica interna del tarugo (DBIT) como variables regresoras, con resultados estadísticos menos satisfactorios que los obtenidos en la tabla 11 .
Luego de este análisis, se optó por el uso del modelo para predecir DBICU con la siguiente expresión.

\section{DBICU $=0,182+0,540 *$ DBMT $-0,008 *(H C *$ DBIT $)$}

CONSIDERACIONES FINALES
- Los tres modelos seleccionados para cada una de las variables respuesta de interés se ajustan adecuadamente.

- Los modelos obtenidos para la parte externa y media del árbol predicen con errores 
mínimos las densidades básicas, siendo menos precisa para la parte interna.

- A bajas densidades, como es el caso de la parte interna árbol el modelo tiene un ajuste menor.

\section{REFERENCIAS}

BARRICHELO, L. E. G. 1979. Estudos das características físicas, anatômicas e químicas da madeira de Pinus caribaea Mor. Var. Hondurensis Berr. E Golf. para a produção de celulose e papel. Piracicaba: 167 p. Tese (Livre Docencia) - ESALQ/USP.

BARRICHELO, L. E. G.; BRITO, J. O. (1978: Manaus), A madeira de Pinus taeda como materia prima para a produção de celulose Kraft, influência da porcentagem de lenho. CONGRESSO FLORESTAL BRASILEIRO Anais. São Paulo: Sociedade Brasileira de Silvicultura, 1978. p. 13 - 18.

BENDTSEN, B. A. 1978. Properties of wood fron improved and intensively managed trees, For Prod. J. v. 28, n. 10, p. 61-72.

C.AR.T.A. 1964 - Compañía Argentina de Relevamientos Topográficos y Aerofotogrametría.

CORONEL, E. O. 1994, Fundamentos de las propiedades físicas y mecánicas de las maderas. v 1.187 p. ITM, Serie de publicaciones 9404. Santiago Del Estero Argentina.

ERIKSON, H. D.; HARRINSON, A. T. 1974. Douglas - fir wood quality studies, Part I Effects of age and stimulate growth on wood density and anatomy. Wood Sci. Technol. v. 8. p. 225-265.

FERREIRA, M. 1970. Estudo da variação da densidade básica de madeira de Eucalyptus alba e Eucalyptus saligna IPEF. Piracicaba: (1): 83-96.

FOELKEL, C. E. B.; BRASIL M. A. M.; BARRICHELO, L. E. G. 1971. Métodos para a determinação da densidade básica para coníferas e latifoliadas. IPEF. v. 2/3, p. 65-74.

HIGA, A. R.; KAGEYAMA, P. Y.; FERRERIRA, M. 1973. Variação da densidade básica de madeira de Pinus elliottii var. Elliottii e Pinus taeda. IPEF, Piracicaba: (7): 79-90.

IFJU, G. 1969. Within growth ring variation in some physical properties of southern pine wood. Wood Sciences. Madison: 2: 11-9.

KNIGGE, W.; SCHULZ, H. 1966. Grundriss der Forestbenutzung Varlag Paul Parey, Hamburg and Berlin. 584 p.

KOLLMANN, F. F. P. 1951. Technologie des Holzes und der Holzwerkstoffe. Band I. Berlin: Springer Verlag,. 1050 p.

KOLLMANN, F. F. P; COTE JUNIOR., W. A. 1968. Principles of wood science and technology. Berlín: Springer, 2 v.

LARSON, P. R. 1969. Wood formation and the concept of wood quality. Bulletin Yale University. School of Forestry. New Haven: (74): 1-53.

MEGRAW, R. A.. 1985. Wood quality factors in Loblolly pine. Atlanta: TAPP PRESS. 88 p.

MENDONZA, M. A. 1982. Características da madeira de Pinus elliottii com diferentes idades e propiedades de papel. Curitiba: 124 f. (Mestrado). Universidade Federal de Paraná. Curso de Engenharia Florestal.

NOAK, D. 1964. "Holzphysik - Vorlesung". Manuskript der Universitat Hamburg.

NYLINDER, P. 1973. Wood quality and fibre products. In: IUFRO MEETING. Proceedings. v. 2. p. $832-844$.

PETERSON, T. A. 1968. Radial variation 1 growth patterns and specific gravity of red pine (Pinus resinosa). For. Prod. J. Madison: 18:62.

PRODEPEF. 1976. (Projeto de desenvolvimento e Pesquisa Florestal). Levantamento da densidade da madeira de Pinus elliottii var. Elliottii, em plantio no sul do Brasil. Série Técnica. $\mathrm{n}^{\mathrm{0}} 5$. PNUD/FAO/IBDF/BRA - 45.

SPURR, S.H.; HSIUNG, W. 1954. Growth rate and specific gravity in conifers $\mathbf{J}$. For., v. 52, n. 3, p. 191 - 192. 
TALBERT, J. T.; JETT, J. B., 1981. Regional specific gravity values for plantation grown, loblolly pine in the Southeastern United States. For. Sci. Madison: 27: $801-7$.

TARAS, M. A., 1965. Some wood properties of slash pine (Pinus elliottii Engelm.) and their relationship to age and heigth within the stem. Agriculture. v. 26, n. 2, p. 559-600.

TRENDELENBUR, R.; MAYER-WEGELIN, H. 1956. Das Holz als Rohstoff. Munchen: Carl Hanser Verlag. 541 p.

YAO, J. 1972. On volumetric shrinkage, specific gravity, and fiber saturation point of loblolly pine wood. Wood Science. Madison: v. 4, p. 171-7.

ZOBEL, B. J.; VAN BUIJTENEN, J. P. 1989. Wood variation. Its causes and control. Springer Verlag. 363 p. 